\title{
FACT - Probing the Periodicity of Mrk 421 \& Mrk 501
}

\author{
M. Beck ${ }^{1, a}$, A. Arbet-Engels ${ }^{1}$, D. Baack ${ }^{2}$, M. Balbo ${ }^{3}$, A. Biland ${ }^{1}$, M. Blank $^{4}$, \\ T. Bretz ${ }^{* 1, a}$, K. Bruegge ${ }^{2}$, M. Bulinski ${ }^{2}$, J. Buss ${ }^{2}$, M. Doerr ${ }^{4}$, D. Dorner ${ }^{4}$, \\ D. Elsaesser ${ }^{2}$, D. Hildebrand ${ }^{1}$, R. lotov $^{4}$, M. Klinger ${ }^{1, a}$, K. Mannheim ${ }^{4}$, S. A. Mueller ${ }^{1}$, \\ D. Neise ${ }^{1}$, A. Neronov ${ }^{3}$, M. Noethe ${ }^{2}$, A. Paravac ${ }^{4}$, W. Rhode ${ }^{2}$, B. Schleicher ${ }^{4}$, \\ K. Sedlaczek ${ }^{2}$, A. Shukla ${ }^{4}$, V. Sliusar ${ }^{3}$, L. Tani $^{1}$, F. Theissen ${ }^{1, a}$, R. Walter ${ }^{3}$ and \\ E. von Willert ${ }^{4}$ (FACT Collaboration')
}

E-mail: marvin.beckerwth-aachen.de, marc.klingererwth-aachen.de, fabian.theissen@rwth-aachen. de

${ }^{1}$ ETH Zurich, Institute for Particle Physics and Astrophysics, Switzerland

${ }^{2}$ TU Dortmund, Experimental Physics 5, Germany

${ }^{3}$ University of Geneva, Department of Astronomy, Switzerland

${ }^{4}$ University of Würzburg, Institute for Theoretical Physics and Astrophysics, Germany

a also at RWTH Aachen University, Physics Institute III A, Germany

\begin{abstract}
Markarian 421 and Markarian 501 are Active Galactic Nuclei and two of the most prominent sources of very high energetic gamma rays. In contrast to the predominantly stable Crab Nebula, the two blazars have extremely variable gamma-ray fluxes. Those could be caused by erratic accretion flow instabilities, but also by processes leading to quasi periodic oscillations (QPOs). Recently, hints of such a QPO of Mrk 501 with a period of about 322 days have been observed with the Large Area Telescope on-board of the Fermi satellite at GeV energies featuring global and local significances of the signal above $99 \%$.
\end{abstract}

The First G-APD Cherenkov Telescope (FACT) has monitored a small sample of known TeV gamma-ray sources including Mrk 421 and Mrk 501 over the last seven years. Therefore, it is ideally suited for periodicity studies of Active Galactic Nuclei, as they require unbiased and continuous long-term observations. Based on measurements of the well-known gamma-ray flux originating from the Crab Nebula, corrections to the measured light curves are applied. In total, 2500 hours of data for each of the two blazars have been analysed using the Lomb-Scargle algorithm. The resulting periodogram of Mrk 501 shows a hint of QPO with a similar period as the one observed by Fermi/LAT.

36th International Cosmic Ray Conference -ICRC2019-

July 24th - August 1st, 2019

Madison, WI, U.S.A.

\footnotetext{
${ }^{*}$ Speaker.

${ }^{\dagger}$ for collaboration list and acknowledgements see PoS(ICRC2019)1177
} 


\section{Introduction}

The First G-APD Cherenkov Telescope (FACT) $[1,2]$ is an Imaging Air Cherenkov Telescope (IACT) pioneering the use of Geiger-mode operated Avalanche Photo Diodes (G-APD, also known as SiPM). Located at the Observatorio del Roque de los Muchachos on the Canary Island of La Palma (Spain), the telescope has been engaging in unbiased long-term monitoring of known $\mathrm{TeV}$ gamma-ray sources since October 2011. The long-term stability and high data-taking efficiency, as well as the ability of the G-APDs to observe even during full moon, make FACT ideally suited for this task. The telescope consists of a camera with 1440 pixels with a total field of view of $4.5^{\circ}$ and a segmented mirror with a total reflective area of $9.5 \mathrm{~m}^{2}$.

The Active Galactic Nuclei (AGN) Mrk 421 and Mrk 501, two of the most prominent sources of gamma rays, have in the past seven years been monitored for more than 2500 hours each [3]. Both blazars are known to exhibit extreme variability, which could be caused by a number of processes. Recently, hints of a quasi periodic oscillation (QPO) of Mrk 501 with a period of about 322 days have been found with Fermi-LAT at $\mathrm{GeV}$ energies featuring global and local significances of the signal above 99\% [4]. Observing such QPOs also in the TeV-range for Mrk 421 or Mrk 501 would lead to a better understanding of the variability of AGN.

\section{Data Reconstruction}

For this study, the data processed off-site with the Modular Analysis and Reconstruction Software (MARS) [5] at the ISDC Data Center for Astrophysics are used. As a first step, the arrival time of the signal in each pixel is extracted and neighbouring pixels with a difference in arrival time below $18.75 \mathrm{~ns} /$ deg are merged into islands. An island survives this image cleaning process, if its signal exceeds 25 pe (photon equivalents). Islands with overlapping arrival time distribution are merged. From the cleaned images, the classical Hillas parameters [6] Width W, Length L, Size $S$ and the center-of-gravity $\vec{P}$ are extracted. In addition, the Slope $\Delta T$ of arrival times along the major axis is calculated.

To ensure that the images contain enough information for a good reconstruction, additional quality cuts are applied: Images after cleaning need to contain more than five pixels and must not consist of more than three distinct islands. The fraction of the signal in the outermost ring of the camera is called Leakage $\ell$. Images having more than $10 \%$ Leakage are discarded.

Using these parameters, the origin of each measured event is reconstructed using the Disp method as described in [7]. The parametrisation of the Disp $P$ was optimised for the smallest relative error on the measured excess using high-quality data from the $\mathrm{TeV}$-standard candle Crab Nebula from the winter of $2017 / 18$ to:

$$
P=\left(1.29^{\circ}+60 \mathrm{ps} \cdot \frac{\Delta T}{\mathrm{~ns} /{ }^{\circ}}+4.26^{\circ} \cdot \ell\right) \cdot\left(1-\frac{W}{L}\right)
$$

Observations are carried out in wobble mode, in which the source is observed off-axis and the background is determined from five positions symmetric around the pointing axis of the telescope. For each of those and the source position, the angular distances $\theta$ to the reconstructed shower origin are calculated respectively. If $\theta<0.19^{\circ}$, the corresponding event is counted as being emitted from this on/off position. 


\section{Data Selection}

The overwhelming majority of measured events are caused by charged cosmic-rays rather than photons. As protons and heavy nuclei in contrast to photons also partake in strong interactions their shower development is characterised by higher transverse momenta. Therefore photon induced showers can be distinguished from cosmic ray induced showers by their narrower lateral distribution. More precisely this is done by comparing the Area A corresponding to the area of an ellipse spanned with the major and minor axis taken as Length and Width with the integral signal (Size) $S$ of an event with the following parametrisation:

$$
\log _{10}\left(\frac{S}{\mathrm{pe}}\right)>1.079 \cdot 10^{-3} \cdot \frac{A}{\mathrm{deg}^{2}}+1.59
$$

The parameters were optimised to obtain the smallest relative error on the integral flux (light curve cut). In fig. 1 this light curve cut is shown. For sake of completeness a different cut for spectral analyses (spectrum cut) is also shown. The variance of the point-spread function obtained for the point-like source signal of measured data of the Crab Nebula is about $0.1^{\circ}$ (see fig. 2).

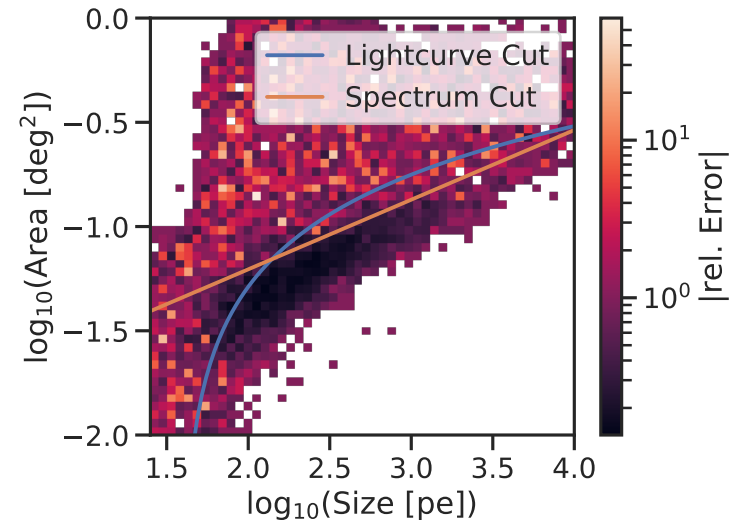

Figure 1: In the background, the relative error on the excess is shown in 2D bins of Area and Size. The light curve cut (blue) removes events which do not fall into the dark region where photon discrimination works best.

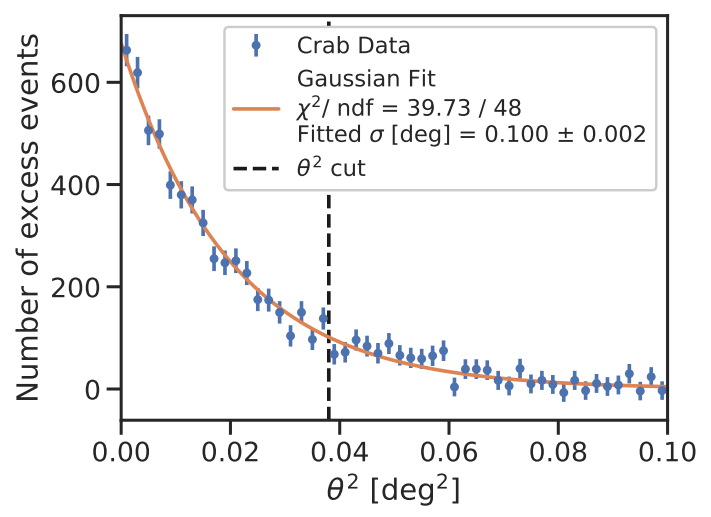

Figure 2: Excess events binned in their reconstructed separation from the on-source position. The distribution (photon point-spread-function) follows a Gaussian shape. The $\theta^{2}$ cut is optimised for the minimal relative error on the excess.

\section{Correcting the Light Curves for Observational Effects}

The measured excess rates are related to the physical flux of a source by a factor. This factor depends on several parameters, such that corrections have to be applied to be able to study the variability of a source's emission. The most dominant effects are the zenith distance of the observation, the light conditions (e.g. moon light) and the atmospheric extinction. As the Crab Nebula is known as the brightest steady source in $\mathrm{TeV}$ astronomy, it can be used to quantify the impact of these effects. In order to allow an isolated investigation of one effect, a strong quality selection on each of the other quantities is applied. In the following, three correction factors are being discussed assuming a spectrum of the Crab Nebula being a power-law with a spectral index of 2.51 in the energy range of interest [9]. 


\subsection{Zenith distance}

Air showers observed at larger zenith angles $\theta$ traverse longer through the atmosphere leading to more scattering and absorption. Additionally, geometric effects spread the photons over a larger area. This leads to lower excess rates $R$. Note that this process is energy dependent and therefore leads to the appearance of the spectral index $\gamma$ in the phenomenological model. The following fit function, with the free parameters $\chi, \kappa$ and $\xi$, is taken from a detailed study in [10]. As the Crab Nebula has not been observed at large zenith distance since winter 2014/2015, the model fit is obtained from data taken only in preceding seasons. The results are shown in fig. 3.

$$
R(\hat{\theta}) \propto \hat{\theta}^{(\gamma-1)(5 \chi+\alpha-2)-2 \chi} \cdot\left(\mathrm{e}^{1-1 / \hat{\theta}}\right)^{\xi(\gamma-1)} \cdot(1-\log \hat{\theta})^{2 \kappa(\gamma-1)} \quad \text { with } \quad \hat{\theta} \equiv \cos \theta .
$$

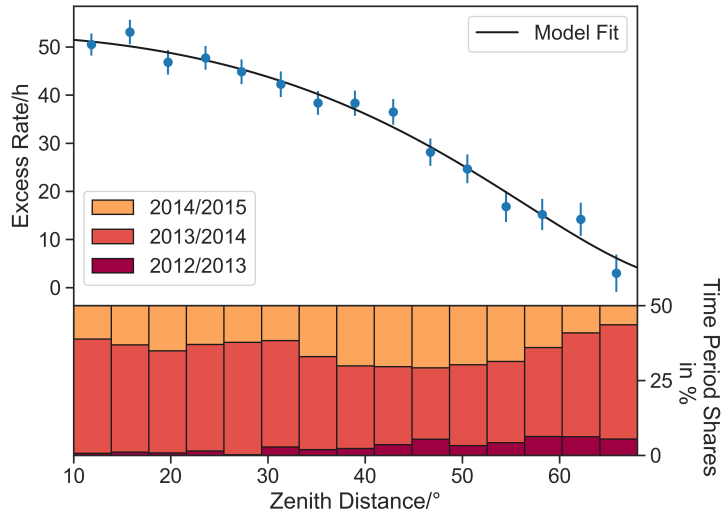

Figure 3: The physically motivated model (eq. 4.1) is fitted to the excess rates of Crab in zenith distance bins. The obtained correction is not biased by contributions of different seasons (see bars below fit).

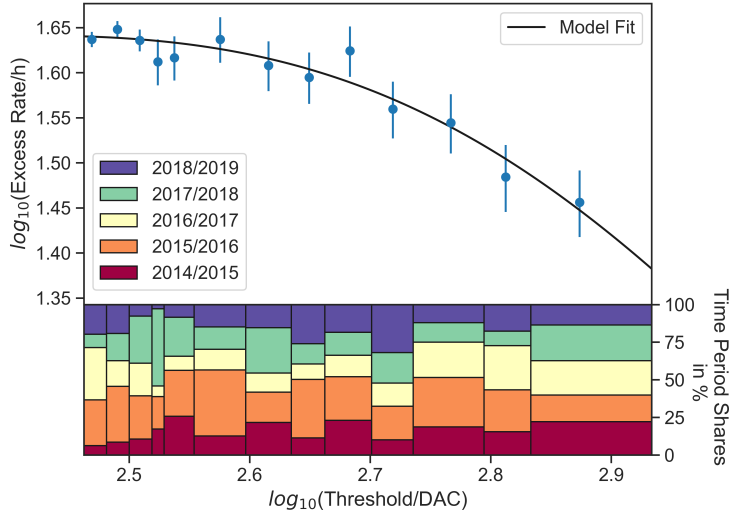

Figure 4: The physically motivated model (eq. 4.2) is fitted to the excess rates of Crab in threshold bins. The obtained correction is not biased by contributions of different seasons (see bars below fit).

\subsection{Trigger threshold}

The threshold $T$ of the hardware trigger is set at the beginning of every data run to account for the changing background noise level, e.g. diffuse moon light (c.f. [2]). Under optimal conditions, the experiment operates at the hardware threshold defined by electronics noise and the lower energy limit of the excess distribution originates from the Area-Size-cut (c.f. sec. 3). As the analysis threshold is higher than the trigger threshold due to the mentioned quality and backgroundsuppression cuts, no dependency of the excess rate from the trigger threshold is expected for lower thresholds. For increasing values of the trigger threshold, the excess rate starts do decrease. Due to the nearly linear correlation between the brightness of extensive air-showers and the primary energy, this corresponds to a proportional increase in the lower energy threshold and consequently leads to a power law with the slope $\gamma+1$ of the measured integral spectrum. This transition is described using a smooth transfer-function $\beta$ (eq. 4.2). The model fit is shown in fig. 4.

$$
R(T) \propto(1-\beta)+\beta \cdot T^{-\gamma+1} \cdot 10^{\mu(\gamma-1)}, \text { with } \beta=\frac{1}{2} \tanh \left(\frac{\log _{10}(T)-\mu}{\sigma}\right)+\frac{1}{2} .
$$




\subsection{Dust concentration}

Due to La Palma's geographical location west of Africa, dust particles from the Sahara can produce a weather phenomenon called Saharan Air-Layer (SAL) or Calima. Its effect on the measured access rate of IACTs was first studied in [11]. A particle counter located at the Telescopio Nationale de Galileo (TNG) measures the dust concentration at about $2360 \mathrm{~m}$. As the SAL typically extends from about $1500 \mathrm{~m}$ to $5500 \mathrm{~m}$, this measurement is assumed to be representative. Although the concentration distribution along the whole shower axis matters for the scattering of Cherenkov photons, the ground layer density correlates well with the total scattering during the passage. Assuming a constant relative loss of photons proportional to the dust concentration $D$ and the total number of photons proportional to their energy, the dependence of trigger and excess rate can be described by:

$$
R(D) \propto(1+\alpha D)^{-\gamma} .
$$

As Calima peaks in summer and the Crab Nebula is only visible in winter, the parameter $\alpha$ is determined using the rate of cosmic ray events obtained with a software trigger as described in [12]. Cross checks with the Crab excess rates for the obtained value of $\alpha$ show a good agreement with the data $\left(\chi^{2} / \mathrm{Ndof}=21.6 / 16\right)$, but the data set is statistically very limited.

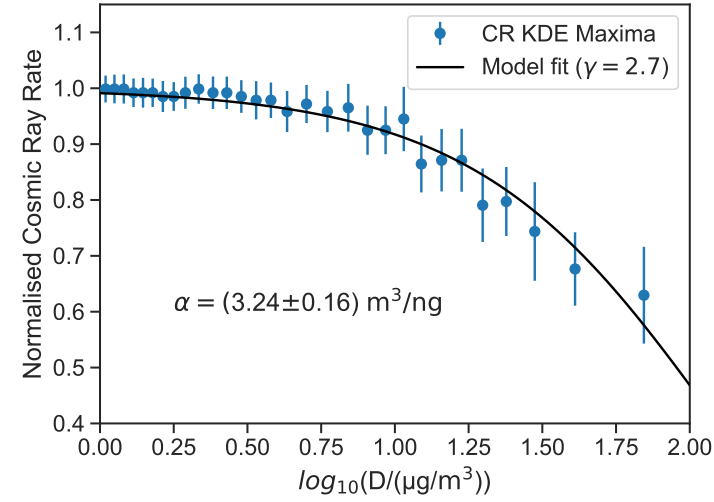

Figure 5: Fit to the normalised cosmic ray rate obtained via a KDE in bins of the dust concentration $D$. The normalisation refers to the average value in pure air.

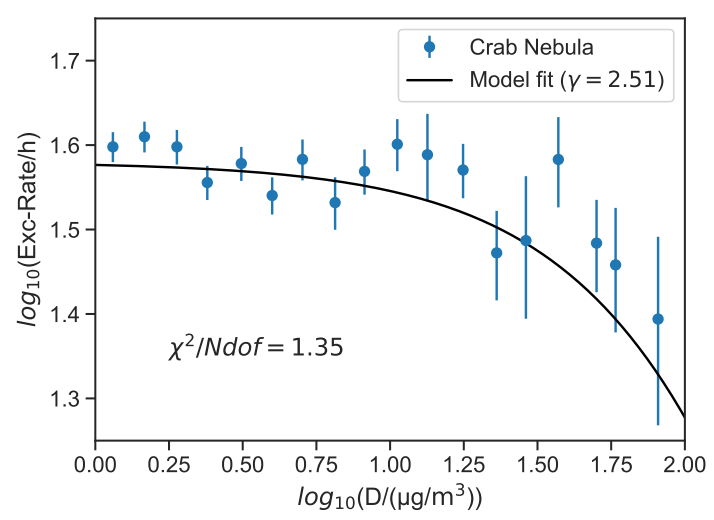

Figure 6: Fit to excess rates of the Crab Nebula with fixed absorption parameter $\alpha$ obtained from the cosmic ray rate dependence (fig. 5).

\subsection{Application of corrections and rebinning}

Data are taken in 5 min runs. Applying run-wise corrections to the measured excess rates is a non-trivial procedure, as signal and background rates are low and the Poissonian nature of the measurement has to be taken into account. Calculating the weighted mean of the excess rates with the correction factor applied run-wise leads to significant underestimations of the measured gammaray flux. Toy Monte Carlo simulations suggest, that for this application the true excess rate can be reconstructed most precisely in the following way: The rates obtained from the individual runs are multiplied with the corresponding correction factors. Then the mean of the results is calculated using only the effective measurement times of the individual runs as weights. The associated Gaussian errors are then added quadratically with the same weights. 


\subsection{Telescope performance correction}

With the above mentioned correction factors applied, the excess rates are still affected by several mechanisms like seasonal variations of the atmosphere or a changing performance of the telescope itself, for example by mirror misalignment or dust on their reflective surfaces. These and other so-far unexplored effects can be taken into account using the correlation to the measured cosmic ray rate, as it is affected in the same way [12]. This yields further, but minor, corrections. Note that mirror misalignments can be monitored independently via their effect on the optical point spread function by analysing the ring-shaped images induced by single muons [8].

\section{Study of Periodicity}

The well established Lomb-Scargle method [13] is used to produce periodograms of the nightlybinned light curves. The periodogram describes how much a periodic oscillation of a given frequency contributes to the light curve. The shape of the resulting periodogram is biased by observational noise and non-uniform sampling of the data points which can introduce artefacts. Especially gaps in the observation ("window function"), for example, due to full moon or the visibility of the source introduce periodic behaviour [14].

To quantify the significance of a frequency, a bootstrapping method is used. A scrambled light curve of a source is produced by randomly reassigning each measurement to the exact time of another measurement. The Lomb-Scargle power is then compared with the $1 \%$ false alarm probability (FAP), which is the 99th percentile of many realisations of scrambled light curves. The
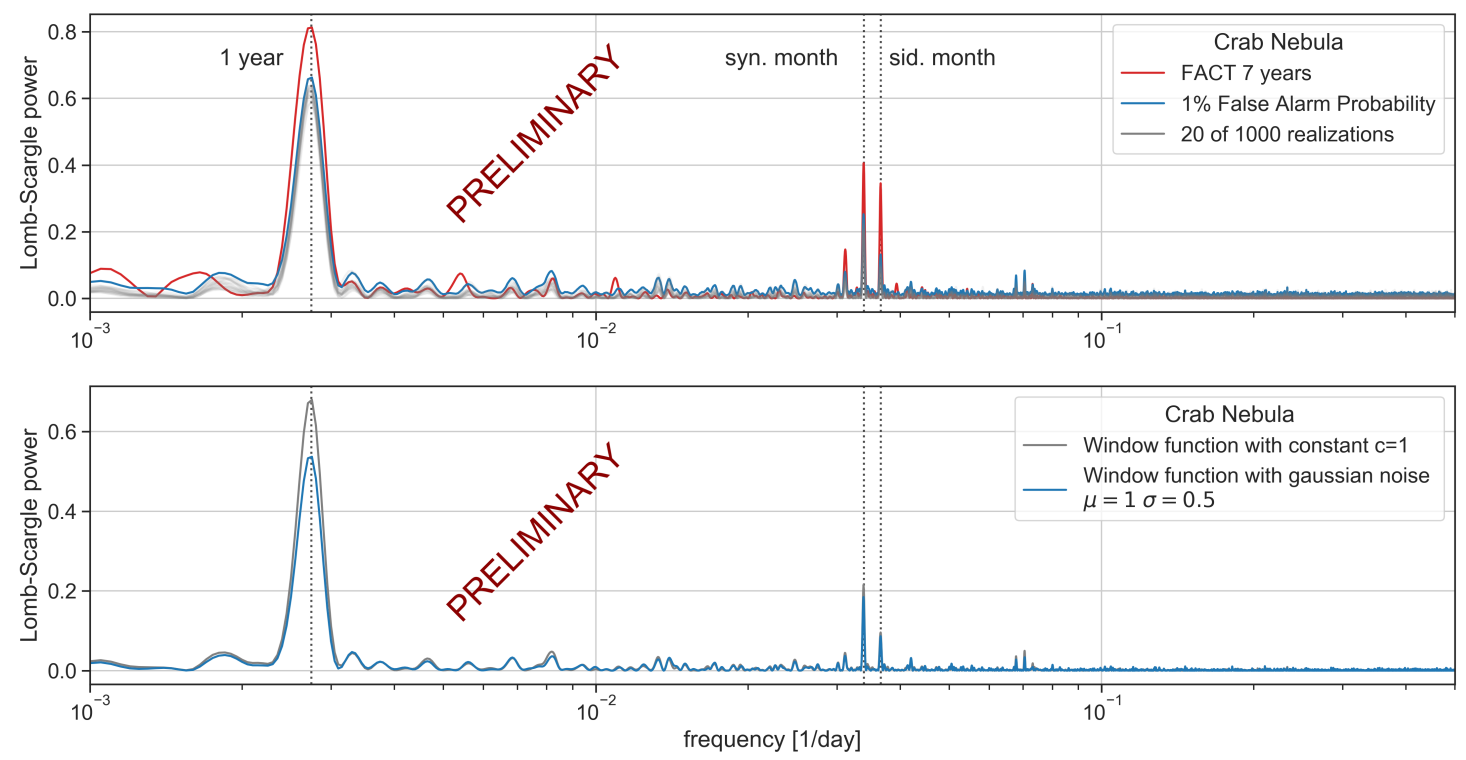

Figure 7: Lomb-Scargle-Periodogram of seven years of FACT data of the Crab Nebula (upper) and the corresponding window function (lower). Also the 1\% false alarm probability and exemplary 20 of 1000 realisations of the scrambled light curves are shown. Three features at periods of one year, as well as the synodal and the sidereal month are marked and arise from the window function. This association is strengthened by the blue curve in the lower plot showing that even a non-constant window function with significant gaussian noise leads to comparable features. 
result is a light curve with the same noise and window function as the original light curve, but excluding any potential periodic behaviour of the source emission. Using many scrambled light curves, a false-alarm-rate is calculated, i.e. the probability that a certain feature of the periodogram is purely the result of noise and the window function.

As an example, fig. 7 shows the application of this method to the Crab Nebula light curve. Two features are clearly visible: A period of one year and two monthly peaks representing the synodal (29.5 days) and the sidereal (27.3 days) moon period, mainly caused by the window-function. Due to aliasing effects these peaks become broadened and sub-peaks are folded in. This indicates that there might be room to further improve the applied corrections, but also that additional effects, such as seasonal changes in the atmosphere, play a role. The results for Mrk 421 and Mrk 501 are shown in fig. 8. For Mrk 501, one additional feature of the periodogram can be seen: a peak at a period of 332 days, corresponding to a period of roughly 322 days in the rest frame of Mrk 501 $\left(z_{M r k 501} \approx 0.034\right)$, which has also been seen by Fermi-LAT [4]. Although broadening leads to an overlap of this peak and the peak for the yearly fluctuations, the 322 days peak is notably absent in the false alarm probability and the periodogram of Mrk 421 which includes very similar systematic effects. Before correcting the effects that lead to the heightened peak for a period of a year, one can not make a quantitative statement how significant the observed oscillation is.
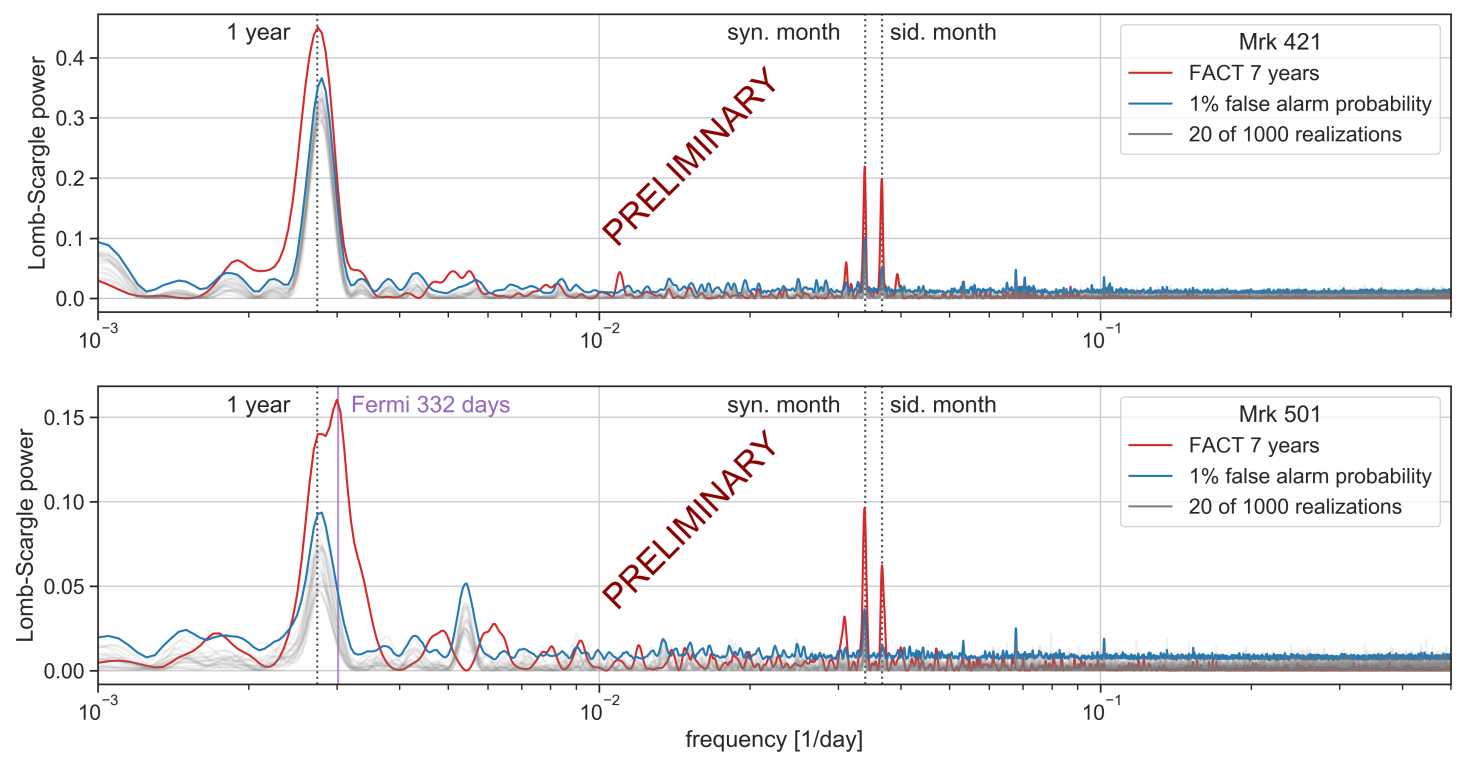

Figure 8: As in fig. 7 the Lomb-Scargle periodograms of Mrk 421 and Mrk 501 with the $1 \%$ false alarm probability and exemplary scrambled realisations are shown. Again, the three features at periods of one year and one month due to the window function are clearly visible. Of particular interest is a new peak for Mrk 501 at a period of 322 days on the falling edge of the peak corresponding to a period of one year.

\section{Conclusion \& Outlook}

The unbiased, densely sampled light curves measured by FACT are ideally suited for studying the variability of blazars in the TeV-range. Several corrections, for the zenith distance of the 
observation, the trigger threshold and the dust concentration of the atmosphere, were derived to produce a corrected light curve that more closely resembles the physical emission of the source. The light curves of the two blazars Mrk 421 and Mrk 501 were investigated for periodic behaviour using the Lomb-Scargle algorithm. The result shows a, as of yet, unaccounted periodic variation with a period of one year in both light curves, potentially caused by atmospheric effects. Additionally, Mrk 501 shows a periodic variation in agreement with a period of 322 days previously seen by Fermi-LAT at lower energies.

As a next step, the effects that produce the currently uncorrected annual variation have to be studied in more detail and corrected for. Then, a quantitative statement on the significance of the observed period can be made.

Acknowledgements: https://fact-project.org/collaboration/icrc2019_acknowledgements.html

\section{References}

[1] H. Anderhub et al. (FACT Collaboration), Design and Operation of FACT - The First G-APD Cherenkov Telescope, JINST Vol. 8 P06008, 2013 [doi:10.1088/1748-0221/8/06/P06008]

[2] A. Biland et al. (FACT Collaboration), Calibration and performance of the photon sensor response of FACT - the first G-APD Cherenkov Telescope, JINST Vol. 9 P10012, 2014 [doi:10.1088/1748-0221/9/10/P10012]

[3] D. Dorner et al. (FACT Collaboration), FACT - Highlights from more than Seven Years of Unbiased Monitoring at TeV Energies, [PoS(ICRC2019)] (in these proceedings)

[4] G. Bhatta, Blazar Mrk 501 shows rhythmic oscillations in its $\gamma$-ray emission, Monthly Notices of the Royal Astronomical Society, May 2019 [arXiv:1808.06067]

[5] T. Bretz and D. Dorner, MARS - CheObs ed. - A flexible Software Framework for future Cherenkov Telescopes, Particle and Space Physics, Detectors and Medical Physics Applications, pp. 681-687, April 2010 [doi:10.1142/9789814307529_0111]

[6] A. M. Hillas. Cerenkov light images of EAS produced by primary gamma, Proceedings of the 19th International Cosmic Ray Conference. Vol. 3, pp. 445-448, 1985

[7] R.W. Lessard et al., A new analysis method for reconstructing the arrival direction of TeV gamma rays using a single imaging atmospheric Cherenkov telscope, APh 15, 2001 [doi:10.1016/S0927-6505(00)00133-X]

[8] Laurits Tani, Monitoring the optical quality of the FACT Cherenkov Telescope, Masterthesis at ETH Zurich, 2019

[9] C. Nigro et al., Towards open and reproducible multi-instrument analysis in gamma-ray astronomy, Astron. Astrophys. Vol. 625, A10, 2019 [doi:10.1051/0004-6361/201834938]

[10] T. Bretz, Zenith angle dependence of the cosmic ray rate as measured with Imaging air-Cherenkov Telescopes Astroparticle Physics, 2019 [doi:10.1016/j.astropartphys.2019.02.004]

[11] D. Dorner et al., A method to correct IACT data for atmospheric absorption due to the Saharan Air Layer, Astron. Astrophys. Vol. 493 p721, 2009 [doi:10.1063/1.3076766]

[12] D. Hildebrand et al. (FACT Collaboration), Using Charged Cosmic Ray Particles to Monitor the Data Quality of FACT [PoS(ICRC2017)779]

[13] M. Zechmeister et al., The generalised Lomb-Scargle periodogram. A new formalism for the floating-mean and Keplerian periodograms, A\&A Vol. 496, pp.577-584, 2009 [doi:10.1051/0004-6361:200811296]

[14] VanderPlas, J. T. Understanding the Lomb-Scargle Periodogram, The Astrophysical Journal Supplement Series, Volume 236, Issue 1, 2018 [doi:10.3847/1538-4365/aab766] 